\title{
Comparative transcriptome analyses of a late-maturing mandarin mutant and its original cultivar reveals gene expression profiling associated with citrus fruit maturation
}

\author{
Wang Lu ${ }^{1,2}$, Hua Qingzhu ${ }^{1}$, Ma Yuewen ${ }^{1}$, Hu Guibing ${ }^{1}$, Qin Yonghua ${ }^{\text {Corresp. }} 1$ \\ ${ }^{1}$ State Key Laboratory for Conservation and Utilization of Subtropical Agro-bioresources/Key Laboratory of Biology and Genetic Improvement of \\ Horticultural Crops-South China, Ministry of Agriculture, College of Horticulture, South China Agricultural University, Guangzhou, China \\ 2 Yunnan Key Laboratory for Wild Plant Resources/Key Laboratory for Economic Plants and Biotechnology, Kunming Institute of Botany, Chinese Academy \\ of Sciences, Kunming, China \\ Corresponding Author: Qin Yonghua \\ Email address: qinyh@scau.edu.cn
}

Characteristics of late maturity in fruits are good agronomic traits for extending the harvest period and marketing time. However, underlying molecular basis of the latematuring mechanism in fruit is largely unknown. In this study, RNA sequencing (RNA-Seq) technology was used to identify differentially expressed genes (DEGs) related to latematuring characteristics from a late-maturing mutant 'Huawan Wuzishatangju' (HWWZSTJ) (Citrus reticulata Blanco) and its original line 'Wuzishatangju' (WZSTJ). A total of approximately $17.0 \mathrm{~Gb}$ and $84.2 \mathrm{M}$ paried-end reads were obtained. DEGs were significantly enriched in the pathway of photosynthesis, phenylpropanoid biosynthesis, carotenoid biosynthesis, chlorophyll and abscisic acid (ABA) metabolism. Thirteen candidate transcripts related to chlorophyll metabolism, carotenoid biosynthesis and ABA metabolism were analyzed using real-time quantitative PCR (qPCR) at all fruit maturing stages of HWWZSTJ and WZSTJ. Chlorophyllase $(C L H)$ and divinyl reductase (DVR) from chlorophyll metabolism, phytoene synthase (PSY) and capsanthin/capsorubin synthase (CCS) from carotenoid biosynthesis, abscisic acid 8'-hydroxylase ( $A B 1)$ and 9-cisepoxycarotenoid dioxygenase (NCED1) from ABA metabolism were cloned and analyzed. The expression pattern of NCED1 indicates its role in the late-maturing characteristics of HWWZSTJ. There were 270 consecutive bases missing in HWWZST] in comparison with fulllength sequences of NCED1 CDNA from WZSTJ. Those results suggested that NCED1 might play an important role in late maturity of HWWZSTJ. This study provides new information of complex process that results in late maturity of Citrus fruit at the transcriptional level. 
1 Comparative transcriptome analyses of a late-maturing mandarin mutant and its original

\section{cultivar reveals gene expression profiling associated with citrus fruit maturation}

${ }^{1}$ State Key Laboratory for Conservation and Utilization of Subtropical Agro-bioresources/Key

Laboratory of Biology and Genetic Improvement of Horticultural Crops-South China, Ministry of Agriculture, College of Horticulture, South China Agricultural University, Guangzhou, China. ${ }^{2}$ Yunnan Key Laboratory for Wild Plant Resources/Key Laboratory for Economic Plants and Biotechnology, Kunming Institute of Botany, Chinese Academy of Sciences, Kunming, China.

\section{Abstract}

Characteristics of late maturity in fruits are good agronomic traits for extending the harvest period and marketing time. However, underlying molecular basis of the late-maturing mechanism in fruit is largely unknown. In this study, RNA sequencing (RNA-Seq) technology was used to identify differentially expressed genes (DEGs) related to late-maturing characteristics from a late-maturing mutant 'Huawan Wuzishatangju' (HWWZSTJ) (Citrus reticulata Blanco) and its original line 'Wuzishatangju' (WZSTJ). A total of approximately 17.0 $\mathrm{Gb}$ and $84.2 \mathrm{M}$ paried-end reads were obtained. DEGs were significantly enriched in the pathway of photosynthesis, phenylpropanoid biosynthesis, carotenoid biosynthesis, chlorophyll and abscisic acid (ABA) metabolism. Thirteen candidate transcripts related to chlorophyll metabolism, carotenoid biosynthesis and $\mathrm{ABA}$ metabolism were analyzed using real-time quantitative PCR (qPCR) at all fruit maturing stages of HWWZSTJ and WZSTJ. Chlorophyllase $(C L H)$ and divinyl reductase $(D V R)$ from chlorophyll metabolism, phytoene synthase (PSY) and capsanthin/capsorubin synthase (CCS) from carotenoid biosynthesis, abscisic acid 8'-hydroxylase $(A B 1)$ and 9-cis-epoxycarotenoid dioxygenase $(N C E D 1)$ from ABA metabolism were cloned and analyzed. The expression pattern of NCEDI indicates its role in the late-maturing characteristics

*Corresponding author. E-mail address: qinyh@scau.edu.cn. 
of HWWZSTJ. There were 270 consecutive bases missing in HWWZSTJ in comparison with full-length sequences of NCED1 cDNA from WZSTJ. Those results suggested that NCED1 might play an important role in late maturity of HWWZSTJ. This study provides new information of complex process that results in late maturity of Citrus fruit at the transcriptional level.

\section{Introduction}

Fruit maturity date is an important economic trait and selection of varieties with different harvest time would be advantageous to extend their storage period and market share. Citrus, one of the most important fruit crops, is large-scale commercial production in the tropical and subtropical regions of the world. The total harvested area of citrus exceeds 8.8 million ha, with an annual yield of over 130 million tons in 2015 (FAOSTAT, 2014). Currently, harvest time for most citrus is mainly from November to December resulting in huge market pressure. Therefore, breeding of early- and late-maturing citrus varieties is essential to extend marketing season, meet the needs of consumers and ensure an optimal adaptation to climatic and geographic conditions.

Plant hormones play important roles in the regulation of fruit development and ripening (Kumar et al., 2014). Ethylene is known to be the major hormonal regulator in climacteric fruit ripening. In addition to ethylene, abscisic acid (ABA), auxin, gibberellin (GA) and brassinosteroid are involved in regulating fruit ripening. ABA plays an important role as an inducer along with ethylene signaling for the onset of fruit degreening and carotenoid biosynthesis during development and ripening process in climacteric and non-climacteric fruits (Leng et al., 2009; Sun et al., 2010; Jia et al., 2011; Romero et al., 2012; Soto et al., 2013; Wang et al., 2016). ABA treatment can rapidly induced flavonol and anthocyanin accumulation in berry skins of the Cabernet Sauvignon grape suggesting that ABA could stimulate berry ripening and ripening-related gene expression (Koyama et al., 2010). ABA also participated in the regulation of fruit development and ripening of tomato (Zhang et al., 2009b; Sun et al., 2011), cucumber (Wang et al., 2013), strawberry (Jia et al., 2011), bilberry (Karppinen et al., 2013), citrus (Zhang 
et al., 2014) and grape (Nicolas et al., 2014). Recent studies showed that ABA is a positive regulator of ripening and exogenous $\mathrm{ABA}$ application could effectively regulate citrus fruit maturation (Wang et al., 2016). Those results suggest that ABA metabolism plays a crucial role in the regulation of fruit development and ripening. In addition, fruit deterioration and post harvest processes might influence fruit quality and ripening process. However, there were few reports involved in those processes. $\alpha$-mannosidase $(\alpha-\mathrm{Man})$ and $\beta$-D-Nacetylhexosaminidase $(\beta-H e x)$ are the two ripening-specific $\mathrm{N}$-glycan processing enzymes have been proved that their transcripts increased with the in non-climacteric fruit ripening and softening (Ghosh et al., 2011). Genetic results have proved that 9-cis-epoxycarotenoid dioxygenase (NCED) is the key enzyme in ABA metabolism in plants (Liotenberg et al., 1999; Luchi et al., 2001). NCED1 could initiate ABA biosynthesis at the beginning of fruit ripening in both peach and grape fruits (Zhang et al., 2009a). Silence of FaNCED1 (encoding a key ABA synthesis enzyme) in strawberry fruit could cause the ABA levels decreased significantly and uncolored fruits and this phenomenon could be rescued by application of exogenous ABA (Jia et al., 2011). Suppression of the expression of SLNCED1 could result in delay of fruit softening and maturation in tomato (Sun et al., 2012). Overexpression of ABA-response element binding factors (SIAREB1) in tomato could regulate organic acid and sugar contents during tomato fruit development. Higher levels of organic acid, sugar contents and related-gene expression were detected in SlAREB1-overexpressing lines in fruit pericarp of mature tomato (Bastías et al., 2014). However, there is little information available about the role of NCED1 genes in citrus fruit maturation (Zhang et al., 2014).

Bud mutant selection is the most common method for creating novel cultivars in Citrus. 'Huawan Wuzishatangju' (HWWZSTJ) mandarin is an excellent cultivar derived from a bud sport of a seedless cultivar 'Wuzishatangju' (WZSTJ). Fruits of HWWZSTJ are mature in late January to early February of the following year, which is approximately $30 \mathrm{~d}$ later than WZSTJ (Qin et al., 2013; Qin et al., 2015). Therefore, the late-maturing mutant and its original cultivar are excellent materials to identify and describe the molecular mechanism involved in citrus fruit 
80

maturation. In this study, the high efficient RNA-Seq technology was used to identify differentially expressed genes (DEGs) between the late-maturing mutant HWWZSTJ and its original line WZSTJ mandarins. DEGs involved in carotenoid biosynthesis, chlorophyll degradation and ABA metabolism were characterized. The present work could help to reveal the molecular mechanism of late-maturing characteristics of citrus fruit at the transcriptional level.

\section{Materials\&Methods}

Plant Materials

The late-maturing mutant 'Huawan Wuzishatangju' (HWWZSTJ) (Citrus reticulata Blanco) and its original cultivar 'Wuzishatangju' (WZSTJ) were planted in the same orchard in South China Agricultural University $\left(23^{\circ} 09^{\prime} 38^{\prime \prime} \mathrm{N}, 113^{\circ} 21^{\prime} 13^{\prime \prime} \mathrm{E}\right)$. Ten six-year-old trees of each cultivar were used in this experiment. Peels (including albedo and flavedo fractions) from fifteen uniform-sized fresh fruits were collected on the $275^{\text {th }}$ (color-break stage, i.e. peels turns from green to orange) and $320^{\text {th }}$ (maturing stage) days after flowering (DAF) of HWWZSTJ an $275^{\text {th }}$ (maturing stage) DAF of WZSTJ ( Fig. S1) in 2012 and pools were named T3, T1 and T2, respectively. Peels from fifteen uniform-sized fresh fruits of HWWZSTJ and WZSTJ were collected on the $255^{\text {th }}, 265^{\text {th }}, 275^{\text {th }}, 285^{\text {th }}, 295^{\text {th }}, 305^{\text {th }}, 315^{\text {th }}$ and $320^{\text {th }}$ DAF in 2012 and used for expression analyses of candidate transcripts associated with chlorophyll, carotenoid biosynthesis and ABA metabolism. All samples were immediately frozen in liquid nitrogen and stored at -80 ${ }^{\circ} \mathrm{C}$ until use.

RNA Extraction, Library Construction and RNA-Seq

Total RNA was extracted from peels according to the protocol of the RNAout kit (Tiandz, Beijing, China) and genomic DNA was removed by DNase I (TaKaRa, Dalian, China). RNA quality was analyzed by $1.0 \%$ agarose gel and its concentration was quantified by a NanoDrop ND1000 spectrophotometer (NanoDrop Technologies, Wilmington, DE, USA). RNA integrity number (RIN) values (>7.0) were assessed using an Agilent 2100 Bioanalyzer (Agilent Technologies, Santa Clara, CA, USA). 
Construction of RNA-Seq libraries was performed by the Biomarker Biotechnology Corporation (Beijing, China). mRNA was enriched and purified with oligo (dT)-rich magnetic beads and then broken into short fragments. The cleaved RNA fragments were reversely transcribed to the first-strand cDNA using random hexamer primers. The second-strand cDNA was synthesized using RNase H and DNA polymerase I. The cDNA fragments were purified, end blunted, 'A' tailed, and adaptor ligated. The distribution sizes of the cDNA in the three libraries were monitored using an Agilent 2100 bioanalyzer. Finally, the three libraries were sequenced using an Illumina $\mathrm{HiSeq}^{\mathrm{TM}} 2500$ platform.

\section{Transcriptome Assembly and Annotation}

Sequences obtained in this study were annotated in reference to the genome sequence of Citrus sinensis (Xu et al., 2013; Wang et al., 2014) using a TopHat program (Trapnell et al., 2009). Functional annotation of the unigenes was performed using BLASTx (Altschul et al., 1997) and classified by Swiss-Prot (SWISS-PROT downloaded from European Bioinformatics Institute by Jan., 2013), Clusters of Orthologous Groups of Proteins Database (COG) (Tatusov et al., 2000), Kyoto Encyclopedia of Genes and Genomes Database (KEGG, release 58) (Kanehisa et al., 2004), non-redundant (nr) (Deng et al., 2006) and Gene Ontology (GO) (Harris et al., 2004). The number of mapped and filtered reads for each unigene was calculated and normalized giving the corresponding Reads Per Kilobases per Million reads (RPKM) values. DEGs between the two samples were determined according to a false discovery rate (FDR) threshold of $<0.01$, an absolute $\log 2$ fold change value of $\geq 2$ and a $\mathrm{P}$-value $<0.01$.

Gene Validation and Expression Analysis

Data from RNA-Seq were validated using qPCR. All pigment-related (chlorophyll metabolism, carotenoid biosynthesis and ABA metabolism) uni-transcripts were selected to elucidate their expression patterns at all peel coloration stages of HWWZSTJ and WZSTJ with specific primers (Table S1). The citrus actin gene (accession No. GU911361.1) was used as an internal standard for the normalization of gene expression. Expression levels of all pigment- 
132 related uni-transcripts were determined using qPCR in an Applied Biosystems 7500 real-time

133 PCR system (Applied Biosystems, CA, USA). A total of $20.0 \mu 1$ reaction volume contained 10.0 $134 \mu$ THUNDERBIRD SYBR qPCR Mix (TOYOBO Co., Ltd.), 50×ROX Reference dye, $2.0 \mu 1$

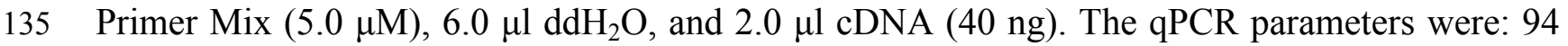
$136{ }^{\circ} \mathrm{C}$ for $60 \mathrm{~s}$ then 40 cycles of $95{ }^{\circ} \mathrm{C}$ for $15 \mathrm{~s}, 55^{\circ} \mathrm{C}$ for $15 \mathrm{~s}$ and $72{ }^{\circ} \mathrm{C}$ for $30 \mathrm{~s}$. All experiments

137 138 139

140

141

142

143

144

145

146

147

148

149

150

151

152

153

154

155

156

157

were performed three times with three biological replicates. Relative expression levels of selected transcripts were calculated by the $2^{-\Delta \Delta C}$ T method (Livak \& Schmittgen., 2001).

All pigment-related genes (chlorophyll metabolism, carotenoid biosynthesis, ABA metabolism) were cloned using specific primers (Table S2). The $20.0 \mu$ l of reaction volume contained $2.0 \mu \mathrm{l} 10 \times \mathrm{PCR}$ buffer, $2.0 \mu \mathrm{l} \mathrm{dNTP}(2.0 \mathrm{mM}), 0.2 \mu \mathrm{l}$ of each primer $(10 \mu \mathrm{M}), 2.0 \mu \mathrm{l}$

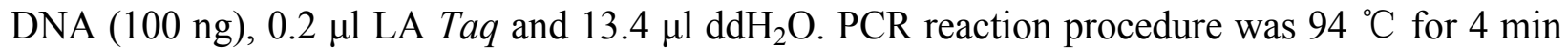
then 35 cycles of $94{ }^{\circ} \mathrm{C}$ for $30 \mathrm{~s}, 55^{\circ} \mathrm{C}$ for $30 \mathrm{~s}$ and $72{ }^{\circ} \mathrm{C}$ for $2 \mathrm{~min}$, with a final $72{ }^{\circ} \mathrm{C}$ for $10 \mathrm{~min}$. Nucleotide sequences of the pigment-related genes were analyzed using the National Center for Biotechnology Information (NCBI) Blast program (http://www.ncbi.nlm.nih.gov/BLAST). ORFs were made using the NCBI ORF Finder (http://www.ncbi.nlm.nih.gov/gorf/gorf.html). Alignments were done using ClustalX 1.83 and DNAMan software. Phylogenetic analysis of deduced amino acid sequences were performed using MEGA (version 5.0) and the Neighborjoining method with 1,000 bootstrap replicates.

\section{Results}

RNA-Seq Analyses

To obtain differentially expressed genes (DEGs) between HWWZSTJ and WZSTJ, three libraries (T1, T2 and T3) were designed for RNA-Seq. As shown in Table 1, 26,403,257, $29,163,126,28,606,868$ raw reads were obtained respectively from the three libraries. After removing low-quality bases and reads, a total of approximately $17.0 \mathrm{~Gb}$ clean reads were obtained. The GC contents for T1, T2 and T3 were $44.27 \%, 44.62 \%$ and $44.20 \%$, respectively 
158 (Table 1). The range of most transcripts length was 100-200 bp (Fig. S2). Q30 percentage 159 (percentage of sequences with sequencing error rate lower than $0.01 \%$ ) for each sample was over $16090 \%$ (Table 1).

161 162

Table 1 Summary of the sequencing data

\begin{tabular}{ccccc}
\hline Samples & Total reads & Total base & GC content $(\%)$ & Q30 (\%) \\
\hline T1 & $26,403,257$ & $5,332,498,617$ & 44.27 & 94.11 \\
T2 & $29,163,126$ & $5,890,197,025$ & 44.62 & 93.98 \\
T3 & $28,606,868$ & $5,777,864,876$ & 44.20 & 93.90 \\
\hline
\end{tabular}

172

Note: T1, HWWZSTJ (320 DAF); T2, WZSTJ (275 DAF); T3, HWWZSTJ (275 DAF).

A total of 44,664,047, 49,507,338 and 48,492,905 reads were mapped which accounted for $84.58 \%, 84.88 \%$ and $84.76 \%$ of the total reads, respectively (Table 2). Number of unique mapped reads accounted for 97.14\% (T1), 97.25\% (T2) and 97.19\% (T3) of the total mapped reads compared with $2.86 \%(\mathrm{~T} 1), 2.75 \%$ (T2) and 2.81\% (T3) for multiple mapped reads, respectively. Those results suggested that the throughput and sequencing quality was high enough for further analyses.

Table 2 Summary of the transcriptome annotation compared with the reference genome of C. Sinensis (Xu et al., 2013)

\begin{tabular}{lcccccc}
\hline \multirow{2}{*}{ Statistics libraries } & \multicolumn{2}{c}{ T1 } & \multicolumn{2}{c}{ T2 } & \multicolumn{2}{c}{ T3 } \\
\cline { 2 - 7 } & Number & Percentage & Number & Percentage & Number & Percentage \\
Total reads & $52,806,514$ & $100.0 \%$ & $58,326,252$ & $100.0 \%$ & $57,213,736$ & $100.0 \%$ \\
Mapped reads & $44,664,047$ & $84.58 \%$ & $49,507,338$ & $84.88 \%$ & $48,492,905$ & $84.76 \%$ \\
Unique mapped reads & $43,386,022$ & $97.14 \%$ & $48,146,871$ & $97.25 \%$ & $47,129,445$ & $97.19 \%$ \\
Multiple mapped reads & $1,278,025$ & $2.86 \%$ & $1,360,467$ & $2.75 \%$ & $1,363,460$ & $2.81 \%$ \\
\hline
\end{tabular}




\begin{tabular}{lcccccc}
\hline Pair mapped reads & $39,251,294$ & $87.88 \%$ & $43,459,426$ & $87.78 \%$ & $42,663,447$ & $87.98 \%$ \\
Single mapped reads & $4,574,673$ & $10.24 \%$ & $5,159,966$ & $10.42 \%$ & $4,969,429$ & $10.25 \%$ \\
\hline
\end{tabular}

175

176

177

178

179

180

181

182

183

184

185

186

187

Note: T1, HWWZSTJ (320 DAF); T2, WZSTJ (275 DAF); T3, HWWZSTJ (275 DAF).

\section{Analyses of Differentially Expressed Genes (DEGs)}

DEGs were screened by comparison between any two of the three libraries using $\mathrm{p} \leq 0.01$ and $2 \leq$ fold changes $\leq 5$ as thresholds. A total of 2,687, 3,002 and 1,834 DEGs were obtained between the $\mathrm{T} 1$ and $\mathrm{T} 3, \mathrm{~T} 2$ and $\mathrm{T} 1, \mathrm{~T} 2$ and $\mathrm{T} 3$ libraries, respectively (Fig. 1A). Among those DEGs, 1,162, 1,567 and 770 were up-regulated and 1,525, 1,435 and 1,064 were down-regulated (Fig. 1B). Transcriptional levels of DEGs in HWWZSTJ on $320^{\text {th }}$ DAF were lower than that on $275^{\text {th }}$ DAF in HWWZSTJ suggesting that transcriptional levels of DEGs decreased during fruit maturation in HWWZSTJ (Fig. 1B).
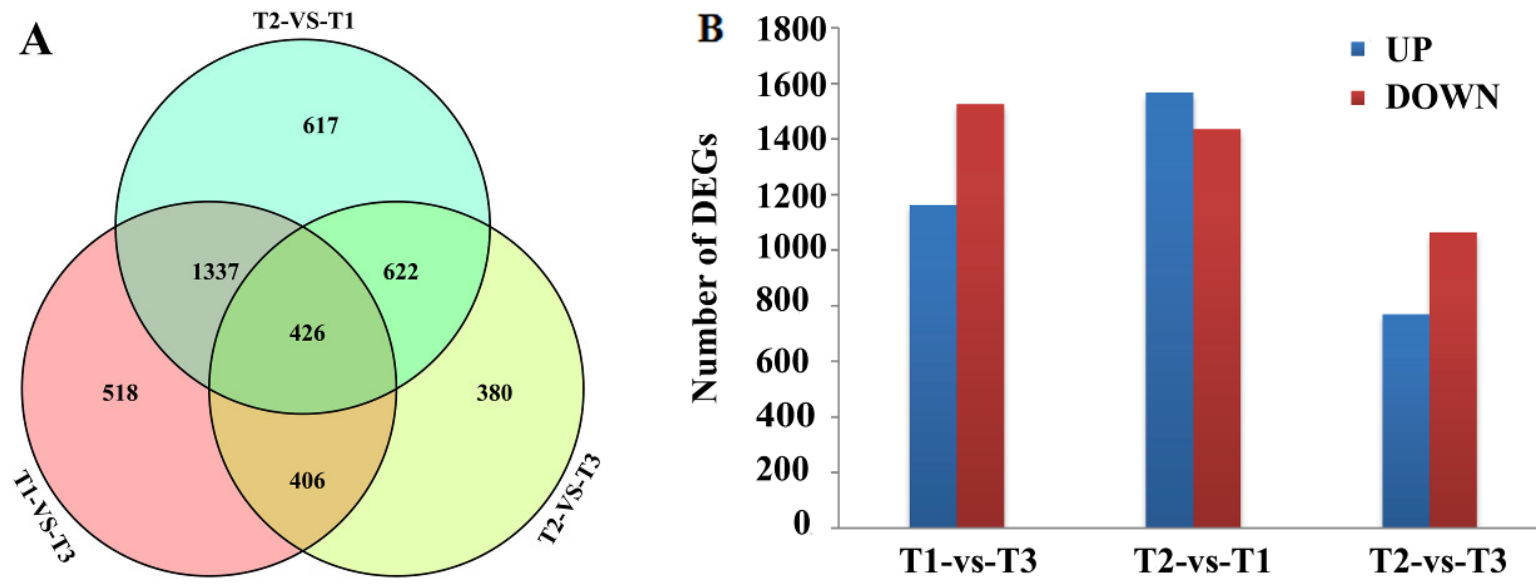

Figure 1 Venn diagram (A) and histogram (B) of DEGs T1, HWWZSTJ (320 DAF); T2, WZSTJ (275 DAF); T3, HWWZSTJ (275 DAF)

Functional Annotation of Transcripts

A total of 299 new transcripts were annotated using five public databases (Nr, Swiss-Prot, KEGG, COG and GO). A summary of the annotations was shown in Table S3. Maximum number of annotation of differentially expressed transcripts $(2,954)$ was $\mathrm{Nr}$ databases by comparison between $\mathrm{T} 1$ and $\mathrm{T} 3, \mathrm{~T} 2$ and $\mathrm{T} 1, \mathrm{~T} 2$ and $\mathrm{T} 3$, followed by GO databases $(2,648)$ 
193 (Table S4). The differentially expressed transcripts were classified into three categories in GO 194 assignments: cellular component, molecular function and biological process. DEGs between T1 195 and T3, T2 and T1, T2 and T3 were all significantly enriched in pigmentation, signaling and 196 growth biological processes (Fig. S3A). Based on COG classifications, differentially expressed 197 transcripts were divided into 25 different functional groups (Fig. S3B). DEGs between any two 198 of the three libraries (T1-VS-T3, T2-VS-T1, T2-VS-T3) were assigned to 91, 100 and 91 KEGG 199 pathways, respectively (File S1), and phenylalanine metabolism, porphyrin and chlorophyll 200 metabolism, and flavonoid biosynthesis were the three most significantly enriched biological 201 processes (Table 3).

202

Table 3 Analyses of differentially expressed transcripts based on KEGG pathway

\begin{tabular}{|c|c|c|c|c|c|c|c|}
\hline \multirow{3}{*}{ \# } & & \multirow{3}{*}{ Pathway } & DEGs with & All genes with & \multirow{3}{*}{ p_value } & \multirow{3}{*}{$\begin{array}{l}\text { corr_p_ } \\
\text { value }\end{array}$} & \multirow{3}{*}{$\begin{array}{c}\text { Pathway } \\
\text { ID }\end{array}$} \\
\hline & & & \multirow{2}{*}{$\begin{array}{c}\text { annotation } \\
\text { (283) }\end{array}$} & \multirow{2}{*}{$\begin{array}{l}\text { annotation } \\
\qquad(3516)\end{array}$} & & & \\
\hline & & & & & & & \\
\hline & 1 & Phenylpropanoid biosynthesis & $24(8.48 \%)$ & $82(2.33 \%)$ & $9.58 \mathrm{e}-09$ & $8.71 \mathrm{e}-07$ & ko00940 \\
\hline & 2 & Photosynthesis & $14(4.95 \%)$ & $46(1.31 \%)$ & $7.90 \mathrm{e}-06$ & $7.19 \mathrm{e}-04$ & ko00195 \\
\hline & 3 & Plant-pathogen interaction & $26(9.19 \%)$ & $130(3.7 \%)$ & $8.30 \mathrm{e}-06$ & $7.56 \mathrm{e}-04$ & ko04626 \\
\hline & 4 & Plant hormone signal transduction & $31(10.95 \%)$ & $180(5.12 \%)$ & $2.73 \mathrm{e}-05$ & $2.49 \mathrm{e}-03$ & ko04075 \\
\hline & 5 & Phenylalanine metabolism & $17(6.01 \%)$ & $72(2.05 \%)$ & $3.59 \mathrm{e}-05$ & $3.26 \mathrm{e}-03$ & ko00360 \\
\hline $\mathrm{T} 1$ & 6 & Photosynthesis-antenna proteins & $7(2.47 \%)$ & $15(0.43 \%)$ & $7.45 \mathrm{e}-05$ & $6.78 \mathrm{e}-03$ & ko00196 \\
\hline vs & 7 & Galactose metabolism & $10(3.53 \%)$ & $45(1.28 \%)$ & $2.46 \mathrm{e}-03$ & $2.23 \mathrm{e}-01$ & ko00052 \\
\hline $\mathrm{T} 3$ & 8 & Starch and sucrose metabolism & $21(7.42 \%)$ & $137(3.9 \%)$ & $2.66 \mathrm{e}-03$ & $2.42 \mathrm{e}-01$ & ko00500 \\
\hline & 9 & $\begin{array}{l}\text { Porphyrin and chlorophyll } \\
\text { metabolism }\end{array}$ & $8(2.83 \%)$ & $37(1.05 \%)$ & $7.83 \mathrm{e}-03$ & $7.12 \mathrm{e}-01$ & ko00860 \\
\hline & 10 & $\begin{array}{l}\text { Amino sugar and nucleotide } \\
\text { sugar metabolism }\end{array}$ & $14(4.95 \%)$ & $89(2.53 \%)$ & $1.06 \mathrm{e}-02$ & $9.66 \mathrm{e}-01$ & ko00520 \\
\hline
\end{tabular}




\begin{tabular}{|c|c|c|c|c|c|c|c|}
\hline & 1 & Photosynthesis & $18(5.17 \%)$ & $46(1.31 \%)$ & $1.1384 \mathrm{e}-07$ & $1.1384 \mathrm{e}-05$ & ko00195 \\
\hline & 2 & Photosynthesis antenna proteins & $10(2.87 \%)$ & $15(0.43 \%)$ & $1.5234 \mathrm{e}-07$ & $1.5234 \mathrm{e}-05$ & ko00196 \\
\hline & 3 & Plant-pathogen interaction & $32(9.2 \%)$ & $130(3.7 \%)$ & $5.5471 \mathrm{e}-07$ & $5.5471 \mathrm{e}-05$ & ko04626 \\
\hline & 4 & Phenylpropanoid biosynthesis & $22(6.32 \%)$ & $82(2.33 \%)$ & $7.9820 \mathrm{e}-06$ & $7.9820 \mathrm{e}-04$ & ko00940 \\
\hline & 5 & Cyanoamino acid metabolism & $9(2.59 \%)$ & $22(0.63 \%)$ & $1.2713 \mathrm{e}-04$ & $1.2713 \mathrm{e}-02$ & ko00460 \\
\hline T2 & 6 & $\begin{array}{l}\text { Biosynthesis of unsaturated } \\
\text { fatty acids }\end{array}$ & $9(2.59 \%)$ & $29(0.82 \%)$ & $1.3656 \mathrm{e}-03$ & $1.3656 \mathrm{e}-01$ & ko01040 \\
\hline \multirow[t]{10}{*}{$\mathrm{T} 1$} & 7 & Phenylalanine metabolism & $16(4.6 \%)$ & $72(2.05 \%)$ & $1.3850 \mathrm{e}-03$ & $1.3850 \mathrm{e}-01$ & ko00360 \\
\hline & 8 & Flavonoid biosynthesis & $10(2.87 \%)$ & $37(1.05 \%)$ & $2.3996 \mathrm{e}-03$ & $2.3996 \mathrm{e}-01$ & ko00941 \\
\hline & 9 & Starch and sucrose metabolism & $23(6.61 \%)$ & $137(3.9 \%)$ & $7.1760 \mathrm{e}-03$ & $7.1760 \mathrm{e}-01$ & ko00500 \\
\hline & 10 & $\begin{array}{l}\text { Stilbenoid, diarylheptanoid } \\
\text { and gingerol biosynthesis }\end{array}$ & $5(1.44 \%)$ & $14(0.4 \%)$ & $8.6944 \mathrm{e}-03$ & $8.6944 \mathrm{e}-01$ & ko00945 \\
\hline & 1 & Photosynthesis & $26(10.48 \%)$ & $46(1.31 \%)$ & $5.1027 \mathrm{e}-19$ & $4.6434 \mathrm{e}-17$ & ko00195 \\
\hline & 2 & Photosynthesis-antenna proteins & $11(4.44 \%)$ & $15(0.43 \%)$ & $1.8431 \mathrm{e}-10$ & $1.6772 \mathrm{e}-08$ & ko00196 \\
\hline & 3 & Phenylpropanoid biosynthesis & $24(9.68 \%)$ & $82(2.33 \%)$ & $6.3286 \mathrm{e}-10$ & $5.7590 \mathrm{e}-08$ & ko00940 \\
\hline & 4 & Phenylalanine metabolism & $16(6.45 \%)$ & $72(2.05 \%)$ & $2.6448 \mathrm{e}-05$ & $2.4068 \mathrm{e}-03$ & ko00360 \\
\hline & 5 & Nitrogen metabolism & $9(3.63 \%)$ & $32(0.91 \%)$ & $2.4845 \mathrm{e}-04$ & $2.2609 \mathrm{e}-02$ & ko00910 \\
\hline & 6 & $\begin{array}{l}\text { Flavone and flavonol } \\
\text { biosynthesis }\end{array}$ & $6(2.42 \%)$ & $15(0.43 \%)$ & $3.3744 \mathrm{e}-04$ & $3.0707 \mathrm{e}-02$ & ko00944 \\
\hline vs & 7 & Cyanoamino acid metabolism & $7(2.82 \%)$ & $22(0.63 \%)$ & $5.4299 \mathrm{e}-04$ & $4.9412 \mathrm{e}-02$ & ko00460 \\
\hline $\mathrm{T} 3$ & 8 & $\begin{array}{l}\text { Stilbenoid, diarylheptanoid } \\
\text { and gingerol biosynthesis }\end{array}$ & $5(2.02 \%)$ & $14(0.4 \%)$ & $1.9788 \mathrm{e}-03$ & $1.8007 \mathrm{e}-01$ & ko00945 \\
\hline & 9 & $\begin{array}{l}\text { Glyoxylate and dicarboxylate } \\
\text { metabolism }\end{array}$ & $7(2.82 \%)$ & $28(0.8 \%)$ & $2.6144 \mathrm{e}-03$ & $2.3791 \mathrm{e}-01$ & ko00630 \\
\hline & 10 & Flavonoid biosynthesis & $8(3.23 \%)$ & $37(1.05 \%)$ & $3.5075 \mathrm{e}-03$ & $3.1918 \mathrm{e}-01$ & ko00941 \\
\hline & 11 & $\begin{array}{l}\text { Porphyrin and chlorophyll } \\
\text { metabolism }\end{array}$ & $8(3.23 \%)$ & $37(1.05 \%)$ & $3.5075 \mathrm{e}-03$ & $3.1918 \mathrm{e}-01$ & ko00860 \\
\hline
\end{tabular}




\begin{tabular}{|c|c|c|c|c|c|c|}
\hline 12 & Plant-pathogen interaction & $18(7.26 \%)$ & $130(3.7 \%)$ & $3.9071 \mathrm{e}-03$ & $3.5554 \mathrm{e}-01$ & ko04626 \\
\hline
\end{tabular}

204

205

206

207

208

209

210

211

212

213

214

215

216

217

218

219

220

221

222

223

224

225

226

227

Verification the Accuracy of the RNA-Seq Data Using qPCR

Twelve DEGs with significant difference from the three libraries were selected for verification of RNA-Seq data by qPCR. Linear regression analysis showed an overall correlation coefficient of 0.828 , indicating a good correlation between qPCR results and the transcripts per kilobase million from the RNA-Seq data (Fig. S4).

DEGs Related to Carotenoid Biosynthesis, Chlorophyll and ABA Metabolism

Analyses of the expression data obtained through RNA-Seq revealed that most DEGs were involved in carotenoid biosynthesis, chlorophyll and ABA metabolism. The main transcripts involved in the three pathways were shown in Table 4 and heatmap were made based on transcripts per kilobase million from the RNA-Seq data (Fig. 2). Three transcripts (Cs8g15480, Pheophorbide a oxygenase; Cs5g16830, Chlorophyllase type 0 and Cs3g19690, Chlorophyll synthase) involved in chlorophyll degradation, six transcripts (Cs3g19770, Delta-aminolevulinic acid dehydratase; Cs9g13460, Magnesium-chelatase subunit H; Cs2g05100, Magnesiumchelatase subunit ChlI-1; Cs7g19710, Magnesium-protoporphyrin O-methyltransferase; Cs6g16200, Magnesium-protoporphyrin IX monomethyl ester [oxidative] cyclase 1 and Cs1g06850, Protochlorophyllide reductase A) involved in chlorophyll biosynthesis, five transcripts (Cs6g15910, Phytoene synthase; Orange1.1t02108, phytoene synthase 2; Cs6g13340, Prolycopene isomerase 1; Cs4g14850/Orange1. 1t00772, Capsanthin/capsorubin synthase and Orange_new Gene_1755, Lycopene beta-cyclase) involved in carotenoid biosynthesis and four transcripts (Cs2g03270/Cs5g14370, 9-cis-epoxycarotenoid dioxygenase 2; Cs6g01180, Xanthoxin dehydrogenase; Cs7g14820, Carotenoid cleavage dioxygenase 4a and Cs6g19380, ABA 8\&apos; -hydroxylase) involved in ABA metabolism were obtained (Fig. 2 and Table 4). 

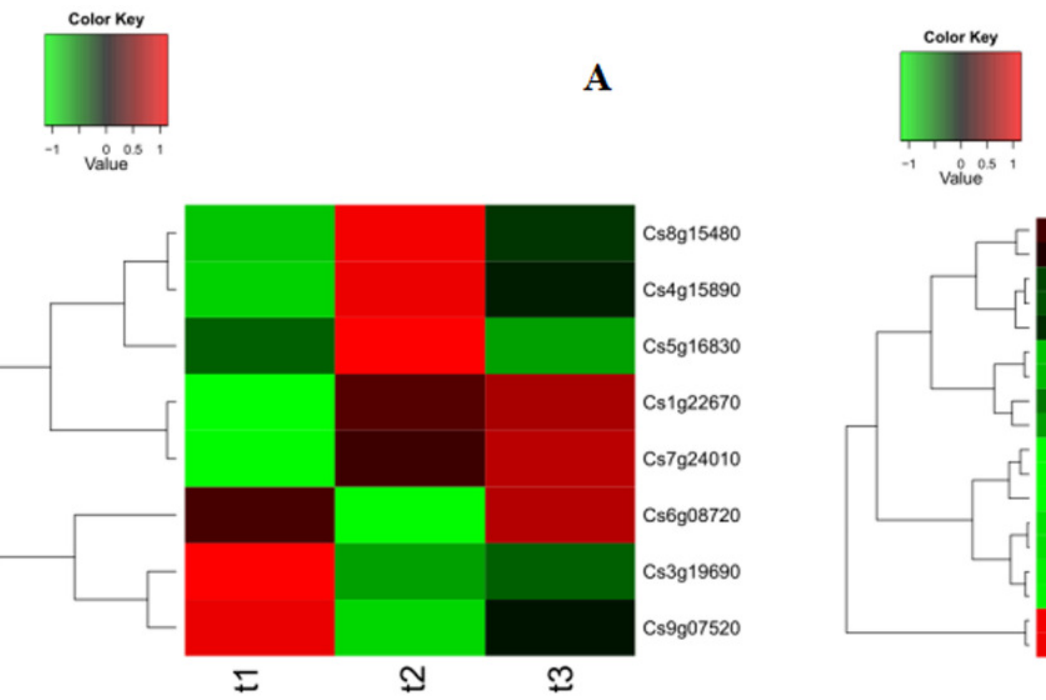

B
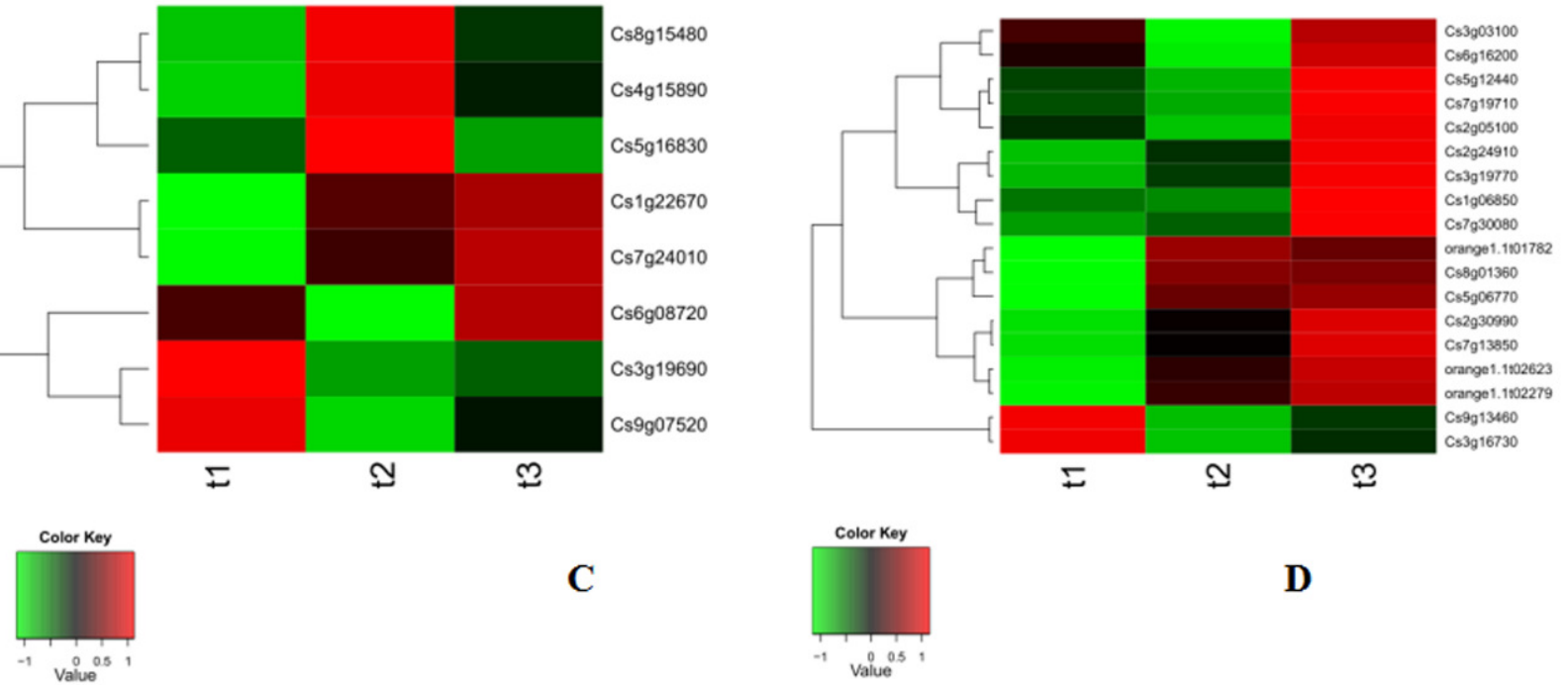

C

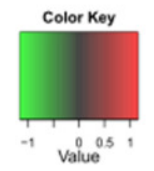

D
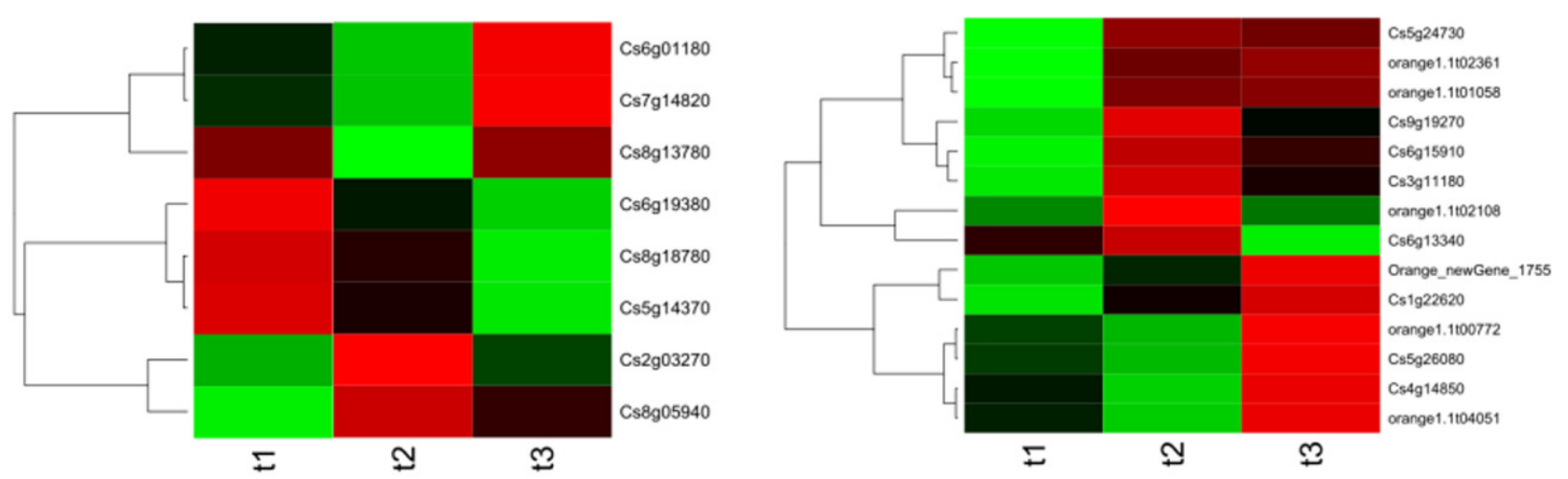

Figure 2 Heatmap of main transcripts from chlorophyll metabolism (A), chlorophyll synthesis

232 Table 4 Analyses of transcripts involved in carotenoid biosynthesis, chlorophyll and ABA 233 metabolism

\section{Gene ID}

RPKM

$\mathrm{T} 1 \quad \mathrm{~T} 2 \quad \mathrm{~T} 3$

Nr-annotation

\section{Chlorophyll metabolism}

$\begin{array}{lllll}\text { Cs3g03100 } & 69.88 & 63.55 & 72.16 & \text { Probable glutamate-tRNA ligase [Arabidopsis thaliana] } \\ \text { Cs } 8 g 01360 & 56.22 & 59.50 & 59.42 & \text { Glutamate-tRNA ligase 1 [Arabidopsis thaliana] }\end{array}$




\begin{tabular}{|c|c|c|c|c|}
\hline Cs3g16730 & 78.03 & 66.88 & 70.76 & Glutamyl-tRNA reductase 1 [Arabidopsis thaliana] \\
\hline Orange1.1t02623 & 89.64 & 138.40 & 164.18 & $\begin{array}{l}\text { Glutamate-1-semialdehyde 2,1-aminomutase 1, } \\
\text { Chloroplastic [Arabidopsis thaliana] }\end{array}$ \\
\hline Cs3g19770 & 35.03 & 42.97 & 62.68 & $\begin{array}{l}\text { Delta-aminolevulinic acid dehydratase, chloroplastic } \\
\text { [Arabidopsis thaliana }]\end{array}$ \\
\hline Cs $7 g 13850$ & 16.13 & 23.66 & 30.83 & Porphobilinogen deaminase [Arabidopsis thaliana] \\
\hline Cs $5 g 12440$ & 21.59 & 19.34 & 27.86 & Uroporphyrinogen decarboxylase 1 , chloroplastic \\
\hline Cs $7 g 30080$ & 53.66 & 58.13 & 83.90 & Uroporphyrinogen decarboxylase 2 , chloroplastic \\
\hline Orange1.1t02279 & 37.48 & 71.77 & 87.12 & Coproporphyrinogen-III oxidase, chloroplastic \\
\hline Cs5g06770 & 4.31 & 6.90 & 7.22 & Oxygen-independent coproporphyrinogen-III oxidase 1 \\
\hline Cs $2 g 24910$ & 21.98 & 25.25 & 31.77 & Protoporphyrinogen oxidase, chloroplastic/mitochondrial \\
\hline Orange1.1t01782 & 35.37 & 56.42 & 53.75 & Protoporphyrinogen oxidase, chloroplastic \\
\hline Cs $9 g 13460$ & 44.52 & 5.36 & 17.65 & Magnesium-chelatase subunit $\mathrm{H}$ \\
\hline Cs $2 g 30990$ & 16.52 & 19.32 & 21.84 & Magnesium-chelatase $67 \mathrm{kDa}$ subunit \\
\hline Cs2g05100 & 118.46 & 82.35 & 183.85 & Magnesium-chelatase subunit ChlI-1, chloroplastic \\
\hline Cs $7 g 19710$ & 6.74 & 3.42 & 18.65 & Magnesium-protoporphyrin O-methyltransferase \\
\hline Cs6g16200 & 76.55 & 14.23 & 116.31 & $\begin{array}{l}\text { Magnesium-protoporphyrin IX monomethyl ester } \\
\text { [oxidative] cyclase } 1\end{array}$ \\
\hline Cs $\lg 06850$ & 22.97 & 15.00 & 167.60 & Protochlorophyllide reductase A, chloroplastic \\
\hline Cs5g 16830 & 5.28 & 29.95 & 0.81 & Chlorophyllase type 0 \\
\hline Cs9g07520 & 30.05 & 13.07 & 20.43 & Chlorophyllase type 0 \\
\hline Cs6g08720 & 57.16 & 45.38 & 61.05 & Bacteriochlorophyll synthase $34 \mathrm{kDa}$ chain \\
\hline Cs3g19690 & 47.34 & 3.63 & 10.36 & Chlorophyll synthase, putative [Ricinus communis] \\
\hline Cs4g15890 & 56.44 & 69.89 & 61.91 & Chlorophyll (ide) b reductase NOL, chloroplastic \\
\hline Cs $7 g 24010$ & 5.24 & 11.18 & 13.54 & Chlorophyll (ide) b reductase NOL, chloroplastic \\
\hline Cs8g15480 & 64.04 & 92.78 & 73.45 & Pheophorbide a oxygenase, chloroplastic \\
\hline Cs $\lg 22670$ & 37.78 & 73.56 & 82.11 & Red chlorophyll catabolite reductase, chloroplastic \\
\hline
\end{tabular}




\section{Carotenoid biosynthesis}

Cs6g15910

Orange1.1t02108

30.15

85.62

32.83

Orange1.1t02361

50.61

63.09

Cs5g24730

47.11

59.11

Cs $3 g 11180$

$56.36 \quad 81.36$

$25.39 \quad 26.28$

Cs6g 13340

Cs4g14850

Orange1.1t00772

7.43

$$
5.56
$$

9.3

8.65

$38.85 \quad 39.93$

Orange1.1t01058

Cs $9 g 19270$

1038.75

Cs 1 g22620

Orange1.1t04051

65.4

1.0

11.76

Cs $5 g 26080$

Orange_new

Gene_1755

\section{ABA metabolism}

Cs8g13780

$4.47 \quad 3.20$

4.5

Cs6g01180

$46.39 \quad 38.09$

Cs $2 g 03270$

Cs5g14370

Cs $7 g 14820$

Cs9g11260

Cs6g19380

Cs8g05940

$\begin{array}{lll}0.45 & 3.82 & 9.60\end{array}$

PREDICTED: phytoene synthase 2, chloroplastic-like [Vitis vinifera]

64.41 Phytoene dehydrogenase, chloroplastic/chromoplastic

58.21 15-cis-zeta-carotene isomerase, chloroplastic

70.96 Phytoene dehydrogenase, chloroplastic/chromoplastic

23.76 Prolycopene isomerase 1, chloroplastic

10.07 Capsanthin/capsorubin synthase, chromoplast

11.12 Capsanthin/capsorubin synthase, chromoplast

39.95 Cytochrome P450 97B1, chloroplastic

1359.83 Beta-carotene 3-hydroxylase 1, chloroplastic

95.98 3-hydroxybenzoate 6-hydroxylase 1

$1.10 \quad 3$-hydroxybenzoate 6-hydroxylase 1

16.31 Violaxanthin de-epoxidase, chloroplastic

$2.08 \quad 67.84 \quad 18.90 \quad 9$ 9-cis-epoxycarotenoid dioxygenase 2 [Citrus sinensis]

Putative 9-cis-epoxycarotenoid dioxygenase 3 [Citrus sinensis]

$\begin{array}{llll}2.18 & 0.26 & 5.90 & \text { Carotenoid cleavage dioxygenase } 4 \mathrm{a} \text { [Citrus clementina] }\end{array}$

$\begin{array}{llll}0.00 & 0.00 & 0.00 & \text { Carotenoid } 9,10\left(9,10^{\prime}\right) \text {-cleavage dioxygenase } 1\end{array}$

$60.57 \quad 30.48 \quad 9.86 \quad$ ABA 8\&apos; -hydroxylase [Citrus sinensis]

$2.05 \quad 3.80 \quad 3.20 \quad$ Abscisic acid 8'-hydroxylase 1 


$\begin{array}{lllll}\text { Cs8g18780 } & 1.10 & 0.78 & 0.27 & \text { ABA 8\&apos; -hydroxylase [Citrus sinensis] }\end{array}$

According to the result of expression and annotation analyses, thirteen transcripts i.e. $B C H P$, CRD1, CHLM, CHLH1, HEMFI/HEMF2, FC1, DVR, CAO, CLH, CCS, PSY, AB and NCED1 associated with chlorophyll metabolism, carotenoid biosynthesis and ABA metabolism were obtained with a Fold Change $\geqq 2$ and FDR $<0.01$ as screening standard (Table 5).

Table 5 Analyses of DEGs associated with carotenoid biosynthesis, chlorophyll and ABA metabolism

\begin{tabular}{ll}
\hline \multicolumn{1}{c}{ Gene ID } & \multicolumn{1}{c}{ Symbols } \\
\hline Chlorophyll metabolism & \\
Cs5g10740/Cs2g26780 & Geranyl acyl geranyl acyl diphosphate reductase $(B C H P)$ \\
Cs6g16200 & Methyl magnesium protoporphyrin IX single cyclase (CRD1) \\
Cs7g19710 & Magnesium protoporphyrin IX methyl transferase $(C H L M)$ \\
Cs2g05100/Cs9g13460 & Mg-chelatase subunit D $(C H L D) /$ Mg-chelatase subunit H $(C H L H 1)$ \\
Orangel1.1t02279 & Coproporphyrin oxidative decarboxylase $(H E M F I / H E M F 2)$ \\
Cs4g18730 & Ferrochelatase $(F C 1)$ \\
Cs1g06850 & Divinyl reductase $(D V R)$ \\
Cs3g19690 & Chlorophyllide a oxygenase $(C A O)$ \\
Cs5g16830 & Chlorophyllase $(C L H)$ \\
Carotenoid biosynthesis & \\
Orange_new Gene_1755 & Capsanthin/capsorubin synthase $(C C S)$ \\
Orange1.1t02108/Cs6g15910 & Phytoene synthase $(P S Y)$ \\
Cs3g23530 metabolism & Abscisic acid 8'-hydroxylase $(A B)$ \\
\hline
\end{tabular}


Expression Analyses of Candidate Transcripts

Expression patterns of candidate transcripts associated with chlorophyll metabolism were analyzed between WZSTJ and HWWZSTJ at all fruit maturation stages (Fig. 3). Compared with WZSTJ, lower expression levels of $A L A D 1$ and $C L H$ were detected in HWWZSTJ at all fruit maturation stages. Expression of $A L A D 1$ and $C L H$ were increasing before fruit maturation and decreased thereafter in both WZSTJ and HWWZSTJ. The highest expression level of $C L H$ was detected on the $295^{\text {th }}$ DAF in HWWZSTJ, which was $20 \mathrm{~d}$ later than WZSTJ. Expression levels of $C A O 1$ and $P A O$ in HWWZSTJ was higher than that in WZSTJ. FC1 showed a decrease trend during fruit maturation of WZSTJ and HWWZSTJ. As for GluRS, CHLH1, HEMG and CHLM, they showed irregular expression patterns in WZSTJ and HWWZSTJ (Fig. 3).
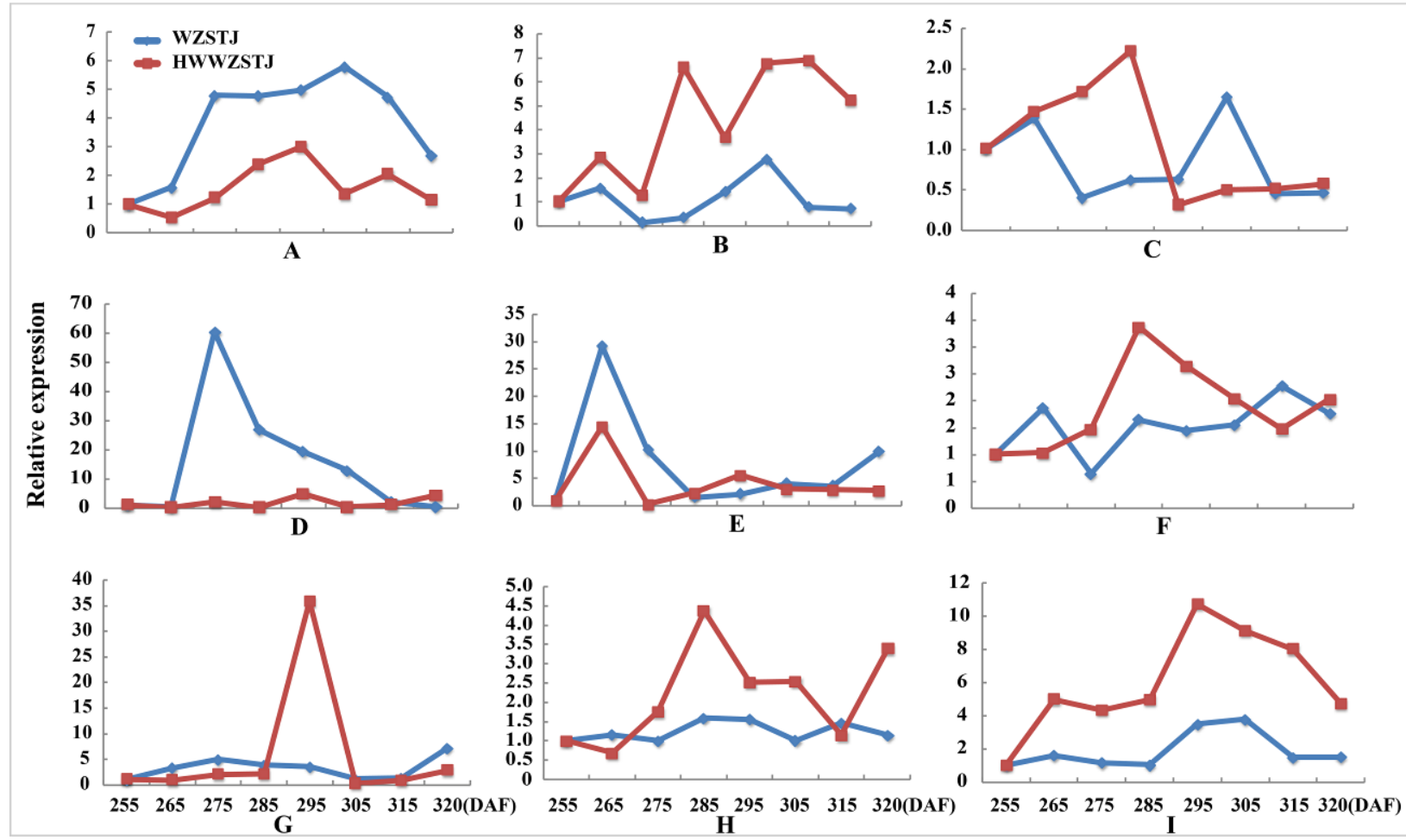

Figure 3 Expression patterns of genes associated with chlorophyll metabolism in WZSTJ and HWWZSTJ at all fruit maturation stages 
Six carotenoid biosynthesis-related transcripts showed a trend from rise to decline at all

261

262

263

264

265

266

267

268

fruit maturation stages of WZSTJ and HWWZSTJ (Fig. 4). The highest expression level of $C C S$ was detected on the $295^{\text {th }}$ DAF in HWWZSTJ which was $20 \mathrm{~d}$ later than that of WZSTJ. Expression levels of PDS1, PSY3, PSY5, PSY6 and PSY7 in WZSTJ were higher than that of HWWZSTJ. PDS1 showed an increasing trend during fruit maturing of WZSTJ and HWWZSTJ and reached its maximum expression on the $295^{\text {th }}$ DAF. PSY5 showed the highest expression levels on the $275^{\text {th }}$ DAF compared to the highest expression levels of PSY3, PSY6 and PSY7 on the 265 ${ }^{\text {th }}$ DAF in both WZSTJ and HWWZSTJ. Expression levels of PSY5 were increasing before the $275^{\text {th }}$ DAF and decreased thereafter. PSY3, PSY6 and PSY7 were up-regulation before the $265^{\text {th }}$ DAF and decreased gradually thereafter (Fig. 4).
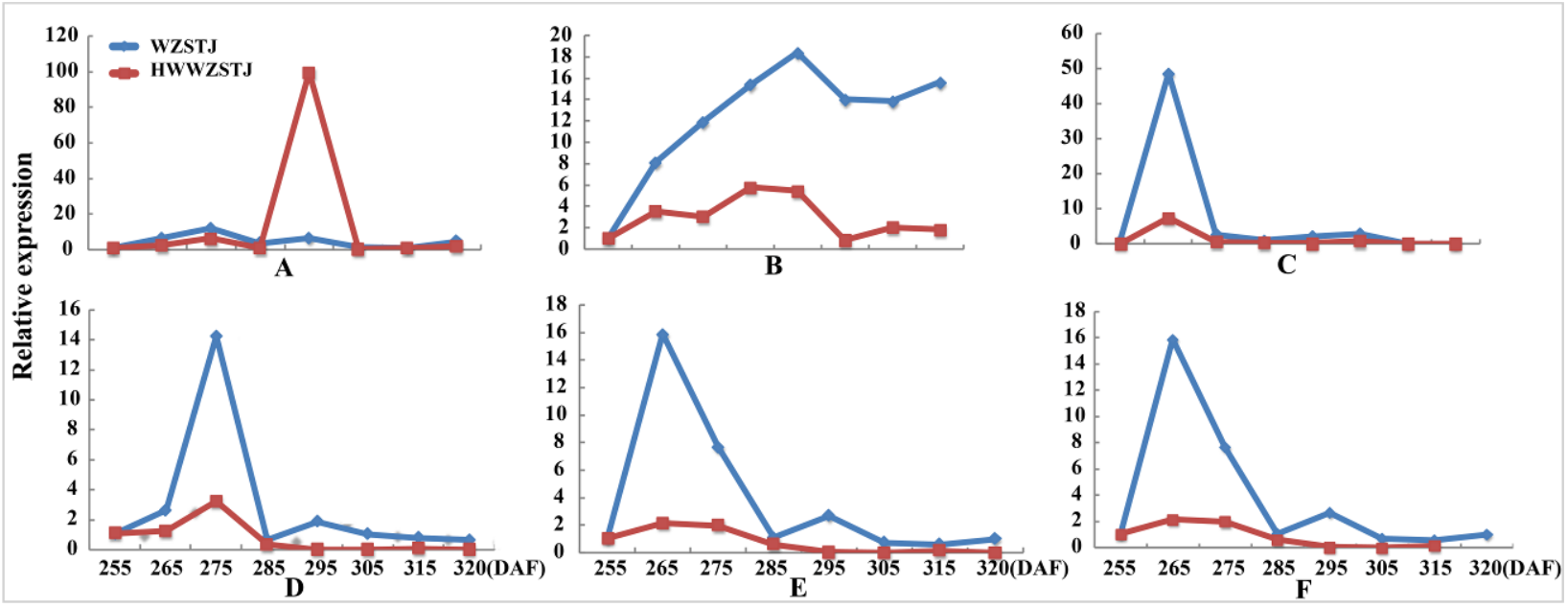

Figure 4 Expression patterns of genes associated with carotenoid biosynthesis in WZSTJ and HWWZSTJ at all fruit maturation stages

A, CCS; B, PDS1; C, PSY3; D, PSY5; E, PSY6; F, PSY7

Expression patterns of two candidate transcripts i.e. $A B 1$ and $N C E D 1$ related to ABA metabolism were analyzed at all fruit maturation stages of WZSTJ and HWWZSTJ (Fig. 5). AB1 
278 showed a trend from rise to decline during fruit maturation stages of WZSTJ and HWWZSTJ. 279 The highest expression level of $A B 1$ was obtained on the $295^{\text {th }}$ DAF in HWWZSTJ, which was $28020 \mathrm{~d}$ later than WZSTJ. Similar expression patterns of NCED1 were observed before the $295^{\text {th }}$ 281 DAF in HWWZSTJ and WZSTJ (Fig. 5). The expression level of NCED1 in HWWZSTJ was 282 lower than that of WZSTJ during $275^{\text {th }}$ DAF to $305^{\text {th }}$ DAF. The highest expression level of 283 NCEDI was detected on the $305^{\text {th }}$ DAF of WZSTJ and significantly deceased thereafter (Fig. 5). 284 However, the highest expression of NCED1 was at 295 DAF in HWWZSTJ. Results from 285 expression analyses of candidate genes suggested that NCED1 might play a leading role in late286 maturing characteristics of HWWZSTJ.
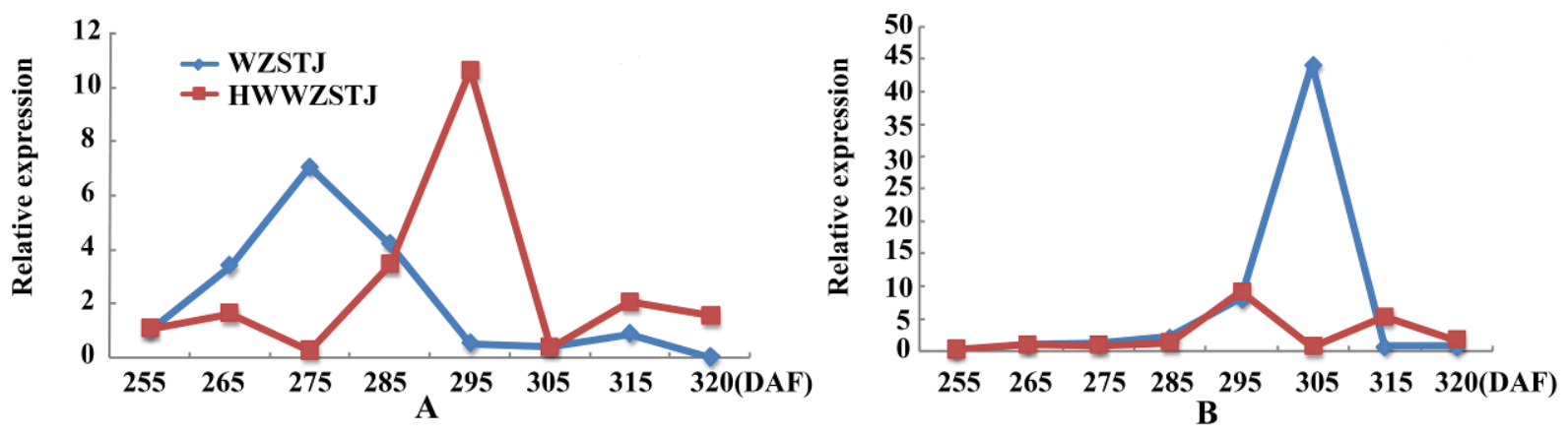

Figure 5 Expression patterns of transcripts associated with ABA metabolism in WZSTJ and HWWZSTJ at all fruit maturation stages

$$
\mathrm{A}, A B 1 ; \mathrm{B}, N C E D 1
$$

Cloning and Phylogenetic Analyses of Candidate Genes

Full-length cDNA sequences of $C L H$ and $D V R$ from chlorophyll metabolism, PSY3, PSY5, PSY6, PSY7 and CCS from carotenoid biosynthesis, AB1 and NCED1 from ABA metabolism were cloned from HWWZSTJ and WZSTJ mandarins. There was one difference in base pair of CLH, PSY3 and PSY5 cDNA sequences between HWWZSTJ and WZSTJ (Fig. S5-7). However, the amino acid sequences of CLH, PSY3 and PSY5 from HWWZSTJ was $100 \%$ identical to that 
300 from WZSTJ. There were 4, 6, 4, 3 and 17 bp difference between the sequences of $D V R, C C S$, $301 \quad P S Y 6, P S Y 7$ and $A B 1$ derived from HWWZSTJ and WZSTJ and this resulted in 2, 3, 3, 1 and 8

302 differences in the amino acids that would have been incorporated during translation of these

303 transcripts (Fig. S8-12). Compared with WZSTJ, there were 270 consecutive bases missing in

304 cDNA sequence of the NCED1 from HWWZSTJ (Fig. 6). Phylogenetic analysis showed that

$305 C L H, D V R, P S Y$ and NCEDI belonged to the same cluster, and their homology in comparison

306 with similar sequences derived from other species is depicted in Fig. S13-16. Results from

307 sequence analyses suggested that deletion of 270 nucleotides in NCED1 maybe result in late-

308 maturing characteristics of HWWZSTJ.

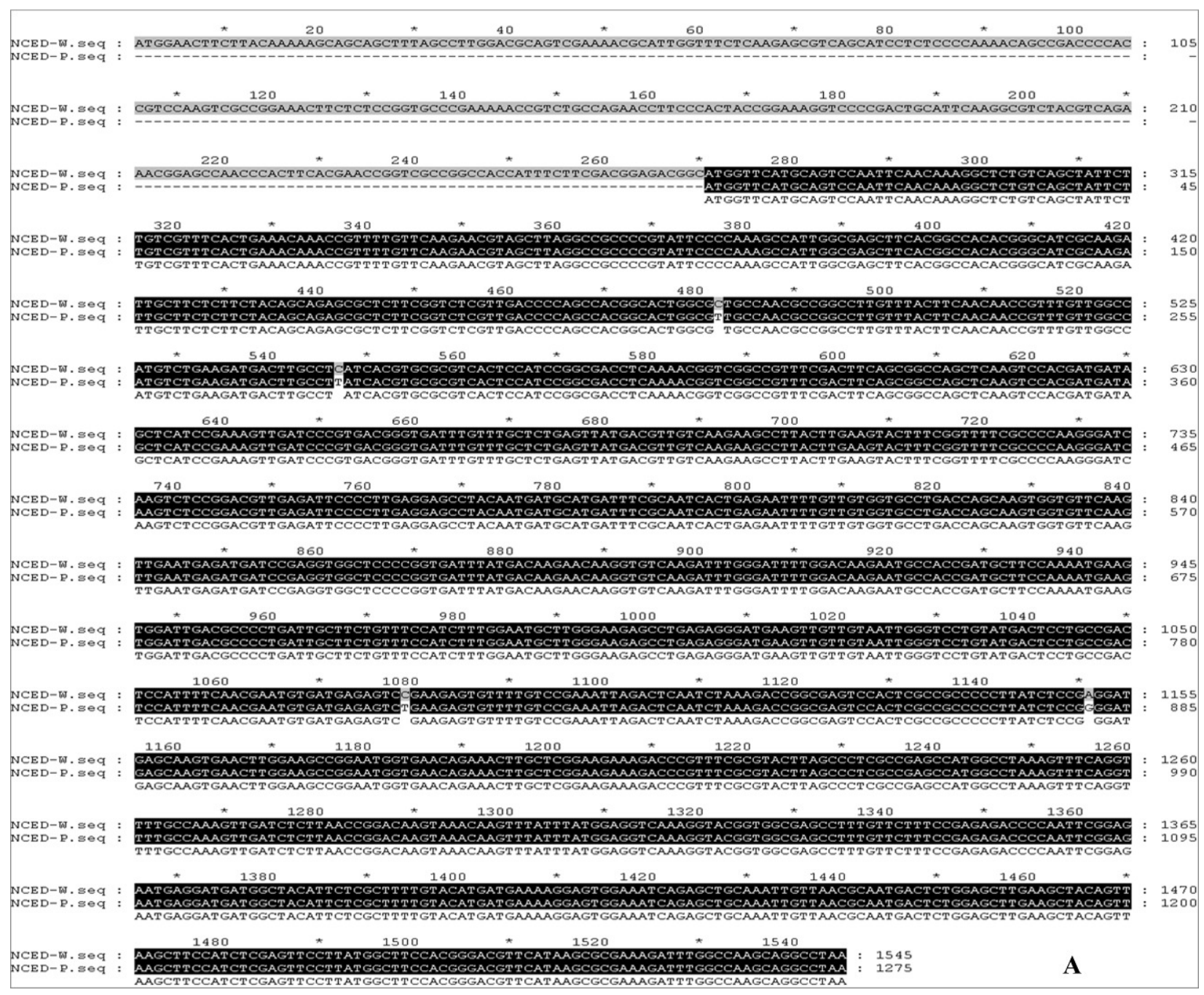




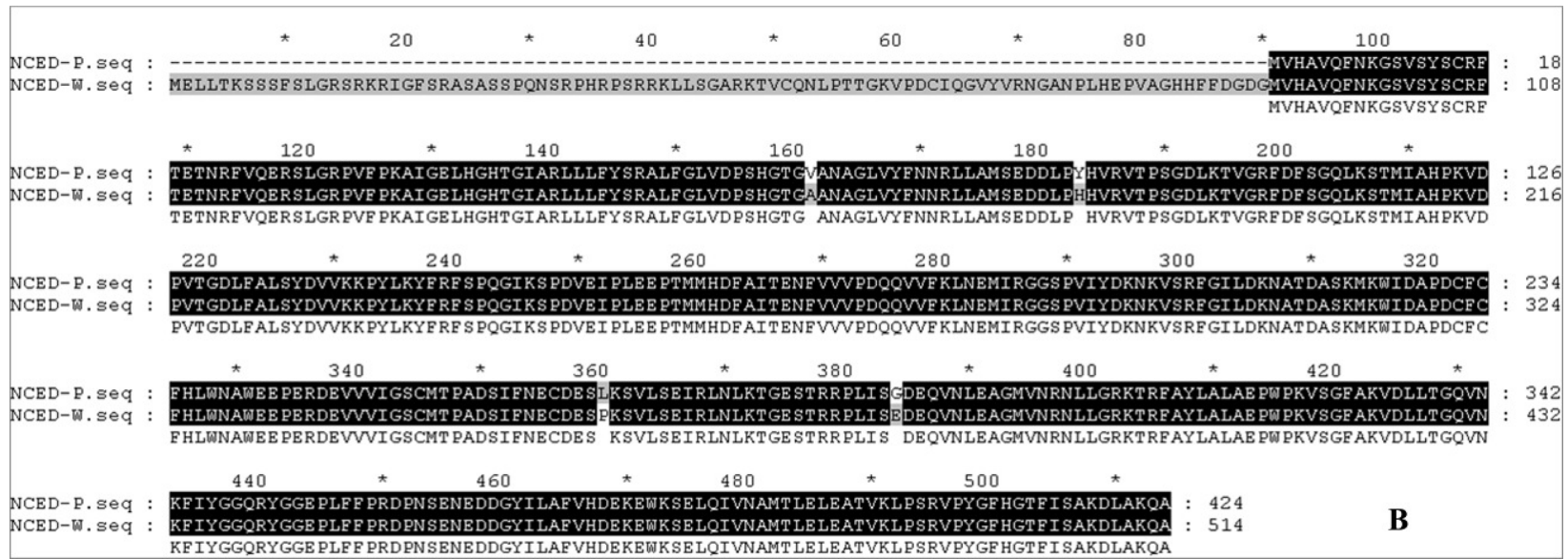

Figure 6 Alignments of cDNA (A) and amino acid (B) sequences of the NCED1 from

HWWZSTJ and WZSTJ

W, HWWZSTJ; P, WZSTJ

\section{Discussion}

Chlorophyll degradation, carotenoid biosynthesis and ABA metabolism play important roles in regulating citrus fruit maturation through a series of related genes or special signal network (Zhang et al., 2014). In this study, RNA-Seq technology was used to screen DEGs between a latematuring mandarin mutant HWWZSTJ and its wild type WZSTJ during fruit maturation. DEGs between any two of the three libraries were significantly enriched in biological processes such as photosynthesis, phenylpropanoid biosynthesis, carotenoid biosynthesis, chlorophyll metabolism, ABA metabolism, starch and sucrose metabolism (Table 3). Thirteen maturing-related genes involved in carotenoid biosynthesis, chlorophyll degradation and ABA metabolism were selected for further analysis.

CLH is the key enzyme catalyzing the first step in the chlorophyll degradation. It can catalyze the hydrolysis of ester bond to yield chlorophyllide and phytol in the chlorophyll breakdown pathway (Jacob-Wilk et al., 1999; Tsuchiya \&Takamiya, 1999). Jacob-Wilk et al

328 isolated a CLH encoding an active chlorophyllase enzyme and verified the role of CLH in 329 chlorophyll dephytylation by in vitro recombinant enzyme assays. Expression level of CLH in 330 Valencia orange peel was low and constitutive and did not significantly increase during fruit 
331 development and ripening (Jacob-Wilk et al., 1999). In the present study, a CLH was obtained 332 from the transcriptome dataset. No difference was detected in the amino acid sequences of CLH 333 between HWWZSTJ and WZSTJ. Expression levels of CLH were increasing prior to citrus fruit 334 maturing, decreasing thereafter in both WZSTJ and HWWZSTJ. The highest expression level of CLH was detected on the $295^{\text {th }}$ DAF in HWWZSTJ, which was $20 \mathrm{~d}$ later than that of WZSTJ (Fig. 3). Similar results were also observed in peels of the late-maturing mutant from Fengjie721 navel orange (Liu et al., 2006) and Tardivo clementine mandarin (Distefano et al., 2009). Those results suggested that CLH may balance between chlorophyll synthesis and its breakdown (Jacob-Wilk et al., 1999).

Citrus is a complex source of carotenoids with the largest number of carotenoids (Kato, 2004). Carotenoid contents and compositions are main factors that affect peel color of most citrus fruits (Tadeo et al., 2008). PSY is a regulatory enzyme in carotenoid biosynthesis (Welsch et al., 2000). $P S Y$ is present at low expression level in unripe (green) melon fruit, reaches its highest levels when the fruit turns from green to orange and persists at lower levels during later ripening stages (Karvouni et al., 1995). Liu et al (2006) studied the mechanism underlying the difference between Fengwan (a late-maturing mutant) navel orange and its original cultivar (Fengjie72-1). The highest expression levels of some carotenoid biosynthetic enzymes in the peels of the late-maturing mutant occurred $30 \mathrm{~d}$ later than that of the original cultivar (Liu et al., 2006). In this work, $P S Y$ showed a trend from rise to decline at all fruit maturation stages of the late-maturing mutant HWWZSTJ and its original line WZSTJ. The expression levels of PSY3, PSY5, PSY6 and PSY7 in HWWZSTJ were lower than that in WZSTJ. These results demonstrated that the mutation in HWWZSTJ influenced the transcriptional activation of PSY.

ABA can be considered as a ripening regulator during fruit maturation and ripening. NCED, a key enzyme involved in ABA biosynthesis, plays an important role in fruit ripening of avocado (Persea americana) (Chernys and Zeevaart, 2007), orange (Citrus sinensis) (Rodrigo et al., 2006), tomato (Solanum lycopersicum) (Nitsch et al., 2009; Zhang et al., 2009b), grape (Vitis vinifera) and peach (Prunus persica) (Zhang et al., 2009a). The NCED1 were expressed only at 
358 the onset stage of ripening in peach and grape, when ABA content became high (Zhang et al., 359 2009a). Zhang et al. (2014) studied the mechanism of a spontaneous late-maturing mutant of 360 'Jincheng' sweet orange and its wild type through the comparative analysis. The highest 361 expression of CSNCED1 was at 215 DAA in WT. In our study, expression levels of NCED1 362 increased prior to fruit maturing and decreased significantly thereafter in both HWWZSTJ and 363 WZSTJ. The highest expression level of NCED1 was detected on the $305^{\text {th }}$ DAF of WT 364 (WZSTJ). Our results were consistent with previous findings that NCED1 plays the most important role in the ABA biosynthesis pathway during the fruit maturing process (Zhang et al., 2014). Deletion of nucleotides could cause a shift of the reading frame and truncated protein, which can result in natural mutants. Compared with cDNA sequence of NCED1 from WZSTJ, there were 270 consecutive bases missing in HWWZSTJ (Fig. 6). Those results suggested that NCED1 might play an important role in late-maturing of HWWZSTJ. A high-efficient regeneration system for WZSTJ has been established (Wang et al., 2015) and further study on the role of NCEDl in citrus is being carried out through genetic engineering.

\section{Conclusion}

RNA-Seq technology was used to identify pigment-related genes from a late-maturing mandarin mutant HWWZSTJ and its original cultivar WZSTJ. Thirteen candidate transcripts related to chlorophyll metabolism, carotenoid biosynthesis and ABA metabolism were obtained. NCED1, a gene involved in ABA metabolism, is probably involved in the formation of late maturity of HWWZSTJ based on sequence and expression analyses. The present study opens up a new perspective to study the formation of late maturity in citrus fruit.

\section{Acknowledgements}

We thank Peng Li, Zixing Ye and Jietang Zhao for help and support with field management and technical assistance.

\section{REFERENCES}


BLAST and PSI-BLAST, A new generation of protein database search programs. Nucleic Acids Research 25: 3389-3402.

Bastías, A., López-Climent, M., Valcárcel, M., Rosello, S., Gómez-Cadenas, A., Casaretto, J.A. 2011. Modulation of organic acids and sugar content in tomato fruits by an abscisic acid-regulated transcription factor. Physiologia Plantarum 141: 215-226.

Chernys, J.T.; Zeevaart, J.A.D. 2007. Characterization of the 9-cis-epoxycarotenoid dioxygenase gene family and the regulation of abscisic acid biosynthesis in avocado. Plant Physiology 124: 343-353.

Deng, Y.Y.; Li, J.Q, Wu, S.F.; Zhu Y.P.; Chen, Y.W.; He, F.C. 2006. Integrated nr database in protein annotation system and its localization. Computer Engineering 5: 71-74.

Distefano, G.; Casas, G.; Caruso, M.; Todaro A.; Rapisarda Paolo.; La Malfa S.; Gentile A.; Tribulato E. 2009. Physiological and molecular analysis of the maturation process in fruits of clementine mandarin and one of its late-ripening mutants. Journal of Agricultural and Food Chemistry 57: 7974-7982.

FAOSTAT. 2014. http://faostat.fao.org/site/567/DesktopDefault.aspx?PageID $\$=\$ 567 \#$ ancor.

Ghosh, S.; Meli, V.S.; Kumar, A.; Thakur, A.; Chakraborty, N.; Chakraborty, S.; Datta, A. 2011. The N-glycan processing enzymes $\alpha$-mannosidase and $\beta$-D-N-acetylhexosaminidase are involved in ripeningassociated softening in the non-climacteric fruits of capsicum. Journal of Experimental Botany 62(2): $571-582$

Harris, M.A.; Clark, J.; Ireland, A.; Lomax, J.; Ashburner, M.; Foulger, R.; Eilbeck, K.; Lewis, S.; Marshall, B.; Mungall, C.; Richter, J.; Rubin, G.M.; Blake, J.A.; Bult, C.; Dolan, M.; Drabkin, H.; Eppig, J.T.; Hill, D.P.; Ni, L.; Ringwald, M.; Balakrishnan, R.; Cherry, J.M.; Christie, K.R.; Costanzo, M.C.; Dwight, S.S.; Engel, S.; Fisk, D.G.; Hirschman, J.E.; Hong, E.L.; Nash, R.S.; Sethuraman, A.; Theesfeld, C.L.; Botstein, D.; Dolinski, K.; Feierbach, B.; Berardini, T.; Mundodi, S.; Rhee, S.Y.; Apweiler, R.; Barrell, D.; Camon, E.; Dimmer, E.; Lee, V.; Chisholm, R.; Gaudet, P.; Kibbe, W.; Kishore, R.; Schwarz, E.M.; Sternberg, P.; Gwinn, M.; Hannick, L.; Wortman, J.; Berriman, M.; Wood, V.; de la Cruz, N.; Tonellato, P.; Jaiswal, P.; Seigfried, T.; White, R. 2004. The Gene Ontology (GO) database and informatics resource. Nucleic Acids Research 32: 258-261

Jacob-Wilk, D.; Holland, D.; Goldschmidt, E.E.; Riov, J.; Eyal, Y. 1999. Chlorophyll breakdown by 
413

414

415

416

417

418

419

420

421

422

423

424

425

426

427

428

429

430

431

432

433

434

435

436

437

438

439 chlorophyllase, Isolation and functional expression of the Chlasel gene from ethylene-treated Citrus fruit and its regulation during development. Plant Journal 20: 653-661.

Jia, H.F.; Chai, Y.M.; Li, C.J; Lu, D.; Luo, J.J.; Qin. L.; Shen, Y.Y. 2011. Abscisic acid plays an important role in the regulation of strawberry fruit ripening. Plant Physiology 157 : 188-199.

Kanehisa, M.; Goto, S.; Kawashima, S.; Okuno, Y.; Hattori, M. 2004. The KEGG resource for deciphering the genome. Nucleic Acids Research 32: 277-280.

Karppinen, K.; Hirvela, E.; Nevala, T.; Sipari, N.; Suokas, M.; Jaakola, L. 2013. Changes in the abscisic acid levels and related gene expression during fruit development and ripening in bilberry (Vaccinium myrtillus L.). Phytochemistry 95: 127-134.

Karvouni, Z.; John, I.; Taylor, J.; Watson, C.F.; Turner, A.J.; Grierson, D. 1995. Isolation and characterisation of a melon cDNA clone encoding phytoene synthase. Plant Molecular Biology 27: 11531162.

Kato, M.; Ikoma, Y.; Matsumoto, H.; Kuniga, T.; Nakajima, N.; Yoshida, T.; Yano, M. 2004. Accumulation of carotenoids and expression of carotenoid biosynthetic genes during maturation in citrus fruit. Plant Physiology 134: 824-837.

Koyama, K.; Sadamatsu, K.; Goto-Yamamoto, N. 2010. Abscisic acid stimulated ripening and gene expression in berry skins of the Cabernet Sauvignon grape. Functional \& Integrative Genomics 10: 367381.

Kumar, R.; Khurana, A.; Sharma, A.K. 2014. Role of plant hormones and their interplay in development and ripening of fleshy fruits. Journal of Experimental Botany 65: 4561-4575.

Leng, P.; Zhang, G.L.; Li. X.X.; Wang. L.H.; Zheng, Z.M. 2009. Cloning of 9-cis-epoxycarotenoid dioxygenase (NCED) gene encoding a key enzyme during abscisic acid (ABA) biosynthesis and ABA regulated ethylene production in detached young persimmon calyx. Chinese Science Bulletin 54: 28302838.

Liu, Y.; Tang, P.; Tao, N.; Xu, Q.; Peng S.A.; Deng X.X.; Xiang K.S.; Huang, R.H. 2006. Fruit coloration difference between Fengwan, a late-maturing mutant and its original cultivar Fengjie 72-1 of Navel Orange (Citrus sinensis Osbeck). Zhiwu Shengli yu Fenzi Shengwuxue Xuebao 32: 31-36. 
440

441

442

Liotenberg S, North H, Marion-Poll A. 1999. Molecular biology and regulation of abscisic acid biosynthesis in plants. Plant Physiology and Biochemistry 37: 341-350.

Livak, K.J.; Schmittgen, T.D. 2011. Analysis of relative gene expression data using real-time quantitative PCR and the $2^{-\triangle \Delta C T}$ method. Methods 25: 402-408.

Luchi S1, Kobayashi M, Taji T, Naramoto M, Seki M, Kato T, Tabata S, Kakubari Y, YamaguchiShinozaki K, Shinozaki K. 2001. Regulation of drought tolerance by gene manipulation of 9-cisepoxycarotenoid dioxygenase, a key enzyme in abscisic acid biosynthesis in Arabidopsis. Plant Journal 27: $325-333$.

Nicolas, P.; Lecourieux, D.; Kappel, C.; Cluzet, S.; Cramer G.; Delrot S.; Lecourieux F. 2014. The bZIP transcription factor $V v A B F 2$ is an important transcriptional regulator of ABA-dependent grape berry ripening processes. Plant Physiology 164: 365-383.

Nitsch, L.; Oplaat, C.; Feron, R.; Ma, Q.; Wolters-Arts, M.; Hedden, P.; Mariani, C.; Vriezen, W.H. 2009. Abscisic acid levels in tomato ovaries are regulated by LeNCED1 and SlCYP707A1. Planta 229: $1335-1346$.

Qin, Y.H.; Li, G.Y.; Wang, L.; Jaime, A.; Ye, Z.X; Feng, Q.R.; Hu, G.B. 2015. A comparative study between a late-ripening mutant of citrus and its original line in fruit coloration, sugar and acid metabolism at all fruit maturation stage. Fruits 70: 5-11.

Qin, Y.H.; Ye, Z.X.; Hu, G.B.; Li, G.Y.; Chen, J.Z.; Lin, S.Q. 2013. 'Huawan Wuzi Shatangju', a latematuring mandarin cultivar. Acta Horticulturae Sinica 40:1411-1412.

Rodrigo, M.J.; Alquezar, B.; Zacarías, L. 2006. Cloning and characterization of two 9-cis-epoxycarotenoid dioxygenase genes, differentially regulated during fruit maturation and under stress conditions, from orange (Citrus sinensis L. Osbeck). Journal of Experimental Botany 57: 633-643.

Romero, P.; Lafuente, M.T.; Rodrigo, M.J. 2012. The Citrus ABA signalosome, identification and transcriptional regulation during sweet orange fruit ripening and leaf dehydration. Journal of Experimental Botany 63: 4931-4945.

Soto, A.; Ruiz, K.; Ravaglia, D.; Costa, G.; Torrigiani, P. 2013. ABA may promote or delay peach fruit ripening through modulation of ripening and hormone-related gene expression depending on the 
developmental stage. Plant Physiology and Biochemistry 64: 11-24.

Sun, L.; Sun, Y.F.; Zhang, M.; Wang, L.; Ren, J.; Cui, M.; Wang, Y.P.; Ji, K.; Li, P.; Li, Q.; Chen, P.; Dai, S.J.; Duan, C.R.; Wu, Y.; Leng, P. 2012. Suppression of 9-cis-Epoxycarotenoid dioxygenase, which encodes a key enzyme in abscisic acid biosynthesis, alters fruit texture in transgenic tomato. Plant Physiology 158: 283-298.

Sun, L.; Wang, Y.P.; Chen, P.; Ren, J.; Ji, K.; Li, Q.; Li, P.; Dai, SJ.; Leng, P. 2011. Transcriptional regulation of SIPYL, SIPP2C, and SlSnRK2 gene families encoding ABA signal core components during tomato fruit development and drought stress. Journal of Experimental Botany 62: 5659-5669.

Sun, L.; Zhang, M.; Ren, J.; Qi, J.; Zhang, G.; Leng P. 2010. Reciprocity between abscisic acid and ethylene at the onset of berry ripening and after harvest. BMC Plant Biology 10: 257-268.

Tadeo, F.R.; Cercos, M.; Colmenero-Flores, J.M.; Iglesias, D.J.; Naranjo, M.A.; Rios, G.; Carrera, E.; Ruiz-Rivero, O.; Lliso, I.; Morillon, R.; Ollitrault, P.; Talon, M. 2008. Molecular physiology of development and quality of citrus. Advances in Botanical Research 47: 147-223.

Tatusov, R.; Galperin, M.; Natale, D.; Koonin, E.V. 2000. The COG database, A tool for genome-scale analysis of protein functions and evolution. Nucleic Acids Research 28: 33-36.

Trapnell, C., Pachter, L., Salzberg, S.L. 2009. TopHat: discovering splice junctions with RNA-Seq. Bioinformatics 25: 1105-1111

Tsuchiya, T.; Takamiya, K.I. 1999. Cloning of chlorophyllase, the key enzyme in chlorophyll degradation, finding of a lipase motif and the induction by methyl jasmonate. Proceedings of the National Academy of Sciences of the United States of America 96: 15362-15367.

Wang, L.; Li, P.; Su, C.L.; Ma, Y.W.; Yan, L.; Hu, G.B.; Qin, Y.H. 2015. Establishment of a high-efficient regeneration system for Wuzishatangju. South China Fruits 44: 48-51.

Wang, X.H., Yin, W., Wu, J.X., Chai, L.J., Yi, H.L. 2016. Effects of exogenous abscisic acid on the expression of citrus fruit ripening-related genes and fruit ripenin. Scientia Horticulturae 201: 175-183

Wang J, Chen DJ, Lei Y, Chang JW, Hao BH, Xing F, Li S, Xu Q, Deng XXin, Chen LL. 2014. Citrus sinensis annotation project (CAP): a comprehensive database for sweet orange genome. PLoS ONE 9: e87723. 
494

495

496

497

498

499

500

501

502

503

Wang, Y.P.; Wang, Y.; Ji, K.; Dai, S.J.; Hu, Y.; Sun, L.; Li, Q.; Chen, P.; Sun, Y.F.; Duan, C.R.; Wu, Y.; Luo, H.; Zhang, D.; Guo, Y.D.; Leng, P. 2013. The role of abscisic acid in regulating cucumber fruit development and ripening and its transcriptional regulation. Plant Physiology and Biochemistry 64: 70-79.

Welsch, R.; Beyer, P.; Hugueney, P.; Kleinig, H.; von Lintig, J. 2000. Regulation and activation of phytoene synthase, a key enzyme in carotenoid biosynthesis, during photomorphogenesis. Planta 211: $846-854$.

Xu, Q.; Chen, L.L.; Ruan, X.; Chen, D.; Zhu, A.; Chen, C.; Bertrand, D.; Jiao, W.B.; Hao, B.H.; Lyon, M.P.; Chen, J.; Gao, S.; Xing, F.; Lan, H.; Chang, J.W.; Ge, X.; Lei, Y.; Hu, Q.; Miao, Y.; Wang, L.; Xiao, S.; Biswas, M.K.; Zeng, W.; Guo, F.; Cao, H.; Yang, X.; Xu, X.W.; Cheng, Y.J.; Xu, J.; Liu, J.H.; Luo, O.J.; Tang, Z.; Guo, W.W.; Kuang, H.; Zhang, H.Y.; Roose, M.L.; Nagarajan, N.; Deng, X.X.; Ruan, Y. 2013. The draft genome of sweet orange (Citrus sinensis). Nature Genetics 45: 59-66.

Zhang, M.; Leng, P.; Zhang, G.; Li, X. 2009a. Cloning and functional analysis of 9-cis-epoxycarotenoid dioxygenase (NCED) genes encoding a key enzyme during abscisic acid biosynthesis from peach and grape fruits. Journal of Plant Physiology 166: 1241-1252.

Zhang, M.; Yuan, B.; Leng, P. 2009b. The role of ABA in triggering ethylene biosynthesis and ripening of tomato fruit. Journal of Experimental Botany 60: 1579-1588.

Zhang, Y.; Wang, X.; Wu, J. 2014. Comparative transcriptome analyses between a spontaneous late-ripening sweet orange mutant and its wild type suggest the functions of ABA, sucrose and JA during citrus fruit ripening. PLoS ONE 9: e116056. 